\title{
Corners Are No Barrier to Imaging Tiny Objects
}

\author{
An update to a technique for imaging around corners allows researchers \\ to capture objects out of the line of sight that have a width of a few human \\ hairs, 100 times tinier than previous demonstrations. \\ By Katherine Wright
}

S eeing around corners without using mirrors might seem impossible, but there are methods that researchers can use to build an approximate image in such situations using scattered light. Until now, these so-called non-line-of-sight (NLOS) techniques had a spatial resolution of a few centimeters. By modifying one such method, a team of researchers in China has achieved a 100 -fold improvement on that resolution, allowing them to image features a few hundreds of micrometers across [1].

NLOS methods image hidden objects by capturing photons that travel along indirect paths from the object to a detector. These photons can scatter off multiple surfaces before landing on the NLOS setup. Each scattering path has a different length, leading to a spread in the arrival times of photons at the detector: a length difference of a few hundred micrometers corresponds to a picosecond difference in photon arrival times. As the maximum temporal resolution of previous photon detectors
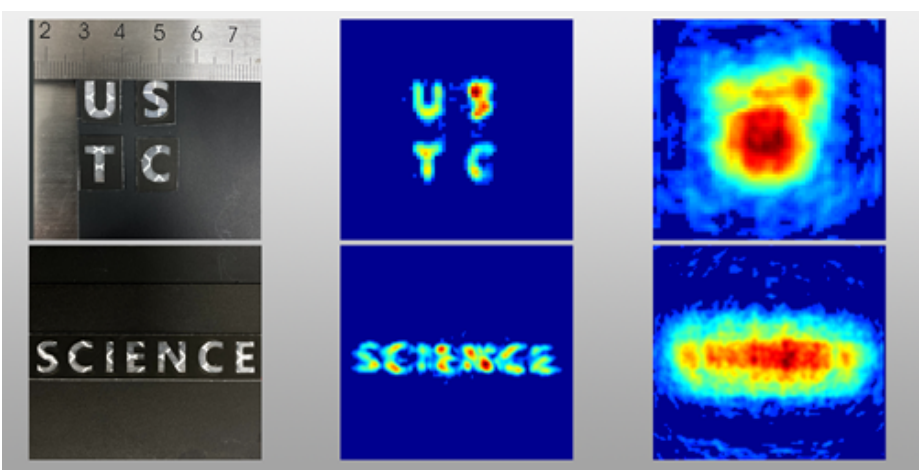

Credit: B. Wang et al. [1] was a few tens of picoseconds, their spatial resolution was limited to a few centimeters.

In their setup, Bin Wang at the University of Science and Technology of China and colleagues replaced the standard photon detector with a laser-pumped "up-conversion" photon detector. The laser pump allowed them to carefully select the photons that registered on the detector and to more precisely determine the arrival times of incoming photons. These two factors, they say, helped to improve the detector's temporal resolution to a picosecond. The team demonstrated the effect of this improvement by using their upgraded setup to image millimeter-high letters hidden behind a wall. The resulting images have an axial resolution of $180 \mu \mathrm{m}$ and a lateral resolution of $2 \mathrm{~mm}$.

Katherine Wright is the Deputy Editor of Physics.

\section{REFERENCES}

1. B. Wang et al., "Non-line-of-sight imaging with picosecond temporal resolution," Phys. Rev. Lett. 127, 053602 (2021). 